\title{
Análise da positividade da esquistossomose mansoni em Regionais de Saúde endêmicas em Pernambuco, 2005 a $2010^{*}$
}

doi: $10.5123 /$ S1679-49742015000100010

\section{Analysis of Schistosomiasis mansoni positivity in endemic Health Regions in the State of Pernambuco, Brazil, 2005-2010}

\author{
Ana Virgínia Matos Sá Barreto \\ Fundação Oswaldo Cruz, Centro de Pesquisas Aggeu Magalhães, Recife-PE, Brasil \\ Secretaria Estadual de Saúde de Pernambuco, Secretaria Executiva de Vigilância em Saúde, Recife-PE, Brasil \\ Nobélia Duarte Melo \\ Universidade de Pernambuco, Faculdade de Enfermagem Nossa Senhora das Graças, Recife-PE, Brasil \\ Jéssica Valença Torres Ventura \\ Universidade de Pernambuco, Faculdade de Enfermagem Nossa Senhora das Graças, Recife-PE, Brasil \\ Rosimery Tavares Santiago \\ Universidade de Pernambuco, Faculdade de Enfermagem Nossa Senhora das Graças, Recife-PE, Brasil \\ Maria Beatriz Araújo Silva \\ Universidade de Pernambuco, Faculdade de Enfermagem Nossa Senhora das Graças, , Recife-PE, Brasil \\ Secretaria Estadual de Saúde de Pernambuco, Laboratório Central de Saúde Pública, Recife-PE, Brasil
}

\section{Resumo}

Objetivo: analisar a tendência da positividade da esquistossomose em exames coproscópicos na população de área endêmica do estado de Pernambuco, Brasil, de 2005 a 2010. Métodos: estudo ecológico de serie temporal, com dados secundários do Sistema de Informação do Programa de Controle da Esquistossomose, analisados por Regional de Saúde; foram avaliadas as tendências por regressão linear, considerando-se $\mathrm{p}<0,05$ significativo. Resultados: Pernambuco apresentou média de 9,2\% de positividade; a Regional de Saúde III (Palmares) apresentou maior média de positividade (13,8\%), seguida das regionais II (Limoeiro: 9,9\%) e I (Recife: 7,8\%); a Regional V (Garanhuns) apresentou melhor média de tratamento (95,6\%), seguida da III (86,6\%); Pernambuco apresentou tendência decrescente na positividade para esquistossomose $(p=0,005)$. Conclusão: em geral, há uma tendência decrescente da positividade de esquistossomose em Pernambuco, apesar da queda no número de exames; é importante manter e intensificar as intervenções de controle, com prioridades estratégicas focalizadas em localidades com elevadas prevalências.

Palavras-chave: Esquistossomose; Pernambuco; Estudos Ecológicos.

\begin{abstract}
Objective: to analyze Schistosomiasis positivity trends in stool tests in the population in endemic area of the state of Pernambuco, Brazil, 2005-2010. Methods: this was an ecological time series study using secondary data from the Schistosomiasis Control Program Information System, analyzed by Health Region. Trends were evaluated using linear regression and $p<0.05$ was considered significant. Results: Pernambuco had average positivity of $9.2 \%$; the $3 r d$ Region (Palmares) had the highest average positivity (13.8\%), followed by the 2nd (Limoeiro: 9.9\%) and the 1st (Recife: $7.8 \%$ ); the 5th Region (Garanhuns) had best average treatment (95.6\%), followed by Palmares (86.6\%); Pernambuco showed a declining trend of Schistosomiasis positivity $(p=0.005)$. Conclusion: in general, there has been a declining trend of Schistosomiasis positivity in Pernambuco, despite the reduction in the number of tests; it is important to maintain and intensify control interventions, with priority strategies focused on highest prevalence locations.
\end{abstract}

Key words: Schistosomiasis; Pernambuco; Ecological Studies.

* Este artigo foi elaborado a partir da monografia de conclusão de curso de graduação em Enfermagem defendida por Nobélia Duarte Melo, Jéssica Valença Torres Ventura e Rosimery Tavares Santiago pela Universidade de Pernambuco (UPE), em 2011.

Endereço para correspondência:

Ana Virgínia Matos Sá Barreto - Programa Sanar, Secretaria Executiva de Vigilância em Saúde, Secretaria Estadual de Saúde de Pernambuco, Rua Dona Maraia Augusta Nogueira, 519, Bongi, Recife-PE, Brasil. CEP: 50751-530

E-mail:aninhabarretoo@gmail.com 


\section{Introdução}

A esquistossomose mansônica é prevalente em regiões tropicais do globo, registrada em 54 países distribuídos pela África, leste do Mediterrâneo e América. Sua magnitude, associada à gravidade das formas clínicas, torna a esquistossomose de grande relevância como problema de Saúde Pública. ${ }^{1}$

Apesar dos esforços para o controle da esquistossomose mansoni, o Brasil é o país da América do Sul onde se concentra o maior número de casos registrados. ${ }^{2}$ No país, a doença ocorre em 19 das 27 unidades federadas, abrangendo uma área endêmica que vai desde o Maranhão até o Espírito Santo e Minas Gerais, além de estar presente, de forma localizada, em outros estados de todas as cinco grandes regiões do país. De acordo com o Ministério da Saúde, em 2010, as prevalências mais elevadas são encontradas nos estados de Alagoas, Pernambuco, Sergipe, Minas Gerais, Bahia, Paraíba e Espírito Santo. ${ }^{3}$ Uma revisão sistemática dos casos de esquistossomose mansoni no Brasil, relativos ao período entre 1997 e 2006, estimou uma prevalência de 2,5 a 6 milhões de indivíduos infectados, 60 a 80\% deles morando na região Nordeste. ${ }^{4}$

\section{Apesar dos esforços para 0 controle da esquistossomose mansoni, - Brasil é o país da América do Sul onde se concentra o maior número de casos.}

Uma avaliação da situação da doença em Pernambuco, no ano de 2010, verificou que a ocorrência da esquistossomose no estado é considerada endêmica em 102 (55,2\%) de seus 185 municípios, principalmente nas regiões da Zona da Mata e Metropolitana do Recife. ${ }^{5}$

Os municípios endêmicos estão localizados em seis das 12 Regionais de Saúde (RS) do estado: RS I; RS II; RS III; RS IV; RS V; e RS XII. O número médio de óbitos anuais em Pernambuco, no período de 2005 a 2010, chegou a 190, com redução na taxa de mortalidade por esquistossomose - por 100 mil habitantes - de 2,29, em 2005, para 2,05, em 2010. Entretanto, Pernambuco apresentou, no mesmo período (2005-2010), a maior taxa de mortalidade por esquistossomose frente à região Nordeste e ao Brasil. ${ }^{5}$
0 atual programa de vigilância e controle dessa endemia no país é o Programa de Controle da Esquistossomose (PCE), o qual inclui atividades de vigilância epidemiológica descentralizadas para o nível municipal nas áreas onde há ocorrência da doença. ${ }^{6} 0$ PCE é executado sobre duas linhas de ação principais, a (i) detecção de portadores, a partir da busca ativa de casos mediante inquéritos coproscópicos realizados com a população, e o (ii) tratamento dos casos positivos, com o objetivo de diminuir a prevalência da infecção e, consequentemente, reduzir a ocorrência das formas graves da doença e a mortalidade a ela associada ${ }^{6}$

A positividade e a distribuição da esquistossomose no estado constituem elementos importantes para o monitoramento de sua tendência. Este trabalho teve como objetivo analisar a tendência da positividade da esquistossomose mansoni detectada pelos exames coproscópicos na população que vive em área endêmica do estado de Pernambuco, Brasil, no período de 2005 a 2010.

\section{Métodos}

Trata-se de uma análise ecológica de série temporal com base em dados secundários, obtidos a partir dos inquéritos coproscópicos realizados na população de 102 municípios considerados endêmicos em Pernambuco. Esses municípios, no período do estudo, estão distribuídos em cinco Regionais de Saúde - RS - e registrados no Sistema de Informação do Programa de Controle da Esquistossomose (SISPCE) da Secretaria Estadual de Saúde de Pernambuco. 0 estudo foi realizado com base no período de 2005 a 2010 por corresponder aos anos utilizados pelo governo de Pernambuco em sua seleção de municípios e localidades prioritárias para o enfrentamento da esquistossomose, por meio do Programa de Enfrentamento às Doenças Negligenciadas (Sanar) no estado de Pernambuco, implantado em 2011. ${ }^{7}$

Pernambuco situa-se na região Nordeste do Brasil. De acordo com o Instituto Brasileiro de Geografia e Estatística (IBGE), sobre dados do Censo Demográfico de $2010,{ }^{8}$ o estado abrange uma área de $98.148,323 \mathrm{~km}^{2}$ e uma população de 8.796 .448 pessoas. Os municípios pernambucanos estão distribuídos em cinco regiões geográficas: Região Metropolitana do Recife, Zona da Mata, Agreste, Sertão e Sertão do São Francisco. Do ponto de vista operacional, para a Secretaria Estadual 
de Saúde, todos esses municípios estão subordinados a 12 RS. Durante a realização deste trabalho, as RS II e XII constituíam apenas uma; porém, no ano de 2012, essas duas RS foram divididas e alguns municípios realocados em outras RS.

A análise dos dados baseou-se no total de exames realizados, total de exames positivos para ovos de Schistosoma mansoni e número de indivíduos tratados no período de 2005 a 2010, em cada RS. A positividade da população estudada corresponde ao quociente entre 0 número de pessoas positivas para S. mansoni e o número total de pessoas examinadas, multiplicado por 100.

A técnica de laboratório recomendada pelo Ministério da Saúde e utilizada pelo PCE durante a realização deste estudo foi o exame coproscópico, segundo o método de Kato-Katz, ${ }^{6}$ considerando-se uma lâmina para cada indivíduo. A cobertura de tratamento corresponde à razão entre o número de tratamentos realizados e o número total de pessoas com exames positivos, multiplicado por 100. Nas ações do PCE, o medicamento preconizado pelo Ministério para 0 tratamento dos casos positivos de esquistossomose é o praziquantel $600 \mathrm{mg}(50 \mathrm{mg} / \mathrm{kg})$, com cobertura de tratamento dos casos positivos acima de $80 \% .{ }^{6}$ As variáveis do estudo foram analisadas para cada RS e para o conjunto do estado de Pernambuco.

Após o levantamento das informações, os dados foram tabulados e analisados pelos programas GraphPad Prism versão 5.01 e Microsoft Office Excel 2010. Analisou-se a tendência para cada RS por regressão linear, onde

\section{$y$ (positividade anual da esquistossomose) $=\alpha+\beta \mathbf{x}$ ano}

e foram consideradas significativas as tendências com $\mathrm{p}<0,05$, sendo também observados o valor do coeficiente de determinação (R2) e a análise dos resíduos. No modelo linear simples, se possuído um conjunto de dados no qual foram medidas duas variáveis, $\mathbf{x}$ e y, cuja correlação entre si indicava um comportamento linear, foi ajustada uma reta que melhor se encaixasse nesses pares de valores pelo método de mínimos quadrados.

A pesquisa foi conduzida conforme a Resolução do Conselho Nacional de Saúde (CNS) n ${ }^{0}$ 466, de 12 de dezembro de 2012, e aprovada pelo Comitê de Ética em Pesquisa da Universidade de Pernambuco mediante o Certificado de Apresentação para Apreciação Ética $\mathrm{n}^{0}$ 0030.0.097.000-11.

\section{Resultados}

De acordo com os dados do SISPCE, foi realizado um total de 1.223.419 exames coproscópicos nos municípios das áreas endêmicas de Pernambuco, entre 2005 e 2010. Desses exames, 120.090 (9,8\%) resultaram positivos para ovos de $S$. mansoni. Entre as RS do estado, a RS III (Palmares) apresentou a maior média de positividade, seguida das RS II (Limoeiro), I (Recife), V (Garanhuns) e IV (Caruaru) (Tabela 1).

Observou-se uma diminuição gradativa nos números de exames coproscópicos realizados no estado, de 279.454 em 2005 para 151.252 em 2008, que voltaram a aumentar nos anos seguintes embora permanecessem abaixo do quantitativo correspondente ao primeiro ano analisado (2005). Tal situação foi especialmente observada na RS III, onde essa redução foi de quase 165 mil exames: ou uma redução proporcional de $88,6 \%$ nos exames realizados, ao se comparar os anos de 2005 e 2010 (Tabela 1).

0 estado de Pernambuco, assim como cada uma das cinco RS analisadas, apresentou tendência decrescente na positividade de exames laboratoriais para a esquistossomose no período de 2005 a 2010. Também foi verificada a presença de tendência temporal significativa de redução da positividade, para o período analisado e em todas as RS, e consequentemente, no estado (Figura 1).

Com relação à cobertura de tratamento dos casos de parasitismo, observou-se uma oscilação entre os anos analisados, nas cinco RS. As RS I (Recife), II (Limoeiro) e IV (Caruaru) apresentaram coberturas de tratamento abaixo do preconizado pelo PCE do Ministério da Saúde. Entretanto, Pernambuco revelou média de cobertura de tratamento de $81,3 \%$ entre os anos de 2005 e 2010 (Tabela 2).

\section{Discussão}

No presente estudo, a média de positividade para esquistossomose em Pernambuco entre 2005 e 2010 foi de $9,2 \%$ - a terceira maior do Brasil -, inferior apenas às dos estados de Alagoas e Sergipe, embora acima da média nacional de 5,9\%, para o mesmo período. ${ }^{9}$ Contudo, a análise de regressão mostrou significativa tendência de redução da positividade para esquistossomose em Pernambuco, como também na ocorrência dos casos por RS. 
Tabela 1 - Número de exames coproscópicos realizados, positivos e positividade para a esquistossomose por Regionais de Saúde (RS) e o conjunto do estado de Pernambuco, 2005 a 2010

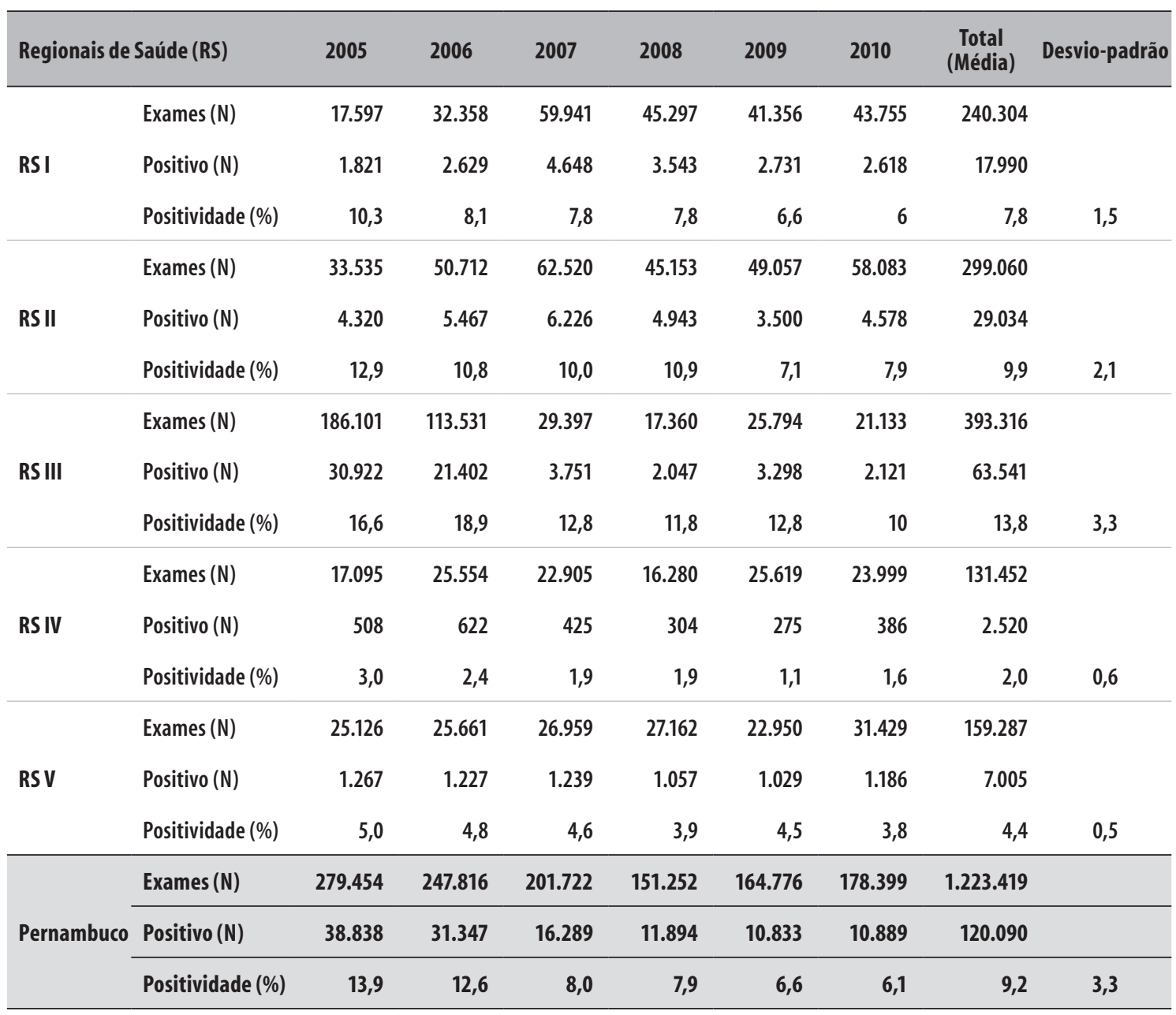

Tabela 2 - Percentual de cobertura de tratamento dos casos positivos por Regionais de Saúde (RS) e o conjunto do estado de Pernambuco, 2005 a 2010

\begin{tabular}{lccccccc}
\hline Regionais de Saúde (RS) & 2005 & 2006 & 2007 & 2008 & 2009 & 2010 & $\begin{array}{c}\text { Média } \\
\text { (Desvio-padrão) }\end{array}$ \\
\hline RSI & 105,3 & 58,6 & 71,6 & 77,6 & 73,8 & 68,1 & $75,8(15,8)$ \\
RS II & 73,0 & 70,4 & 76,0 & 79,9 & 76,3 & 75,7 & $75,2(3,2)$ \\
RS III & 85,2 & 88,0 & 89,4 & 84,3 & 87,4 & 85,5 & $86,6(1,9)$ \\
RS IV & 90,9 & 82,8 & 70,8 & 85,2 & 74,5 & 70,2 & $79,1(8,5)$ \\
RS V & 81,8 & 86,6 & 139,6 & 80,8 & 96,5 & 88,2 & $95,6(22,3)$ \\
\hline Pernambuco & 84,8 & 82,3 & 82,5 & 80,2 & 80,9 & 77,0 & $81,3(2,6)$ \\
\hline
\end{tabular}




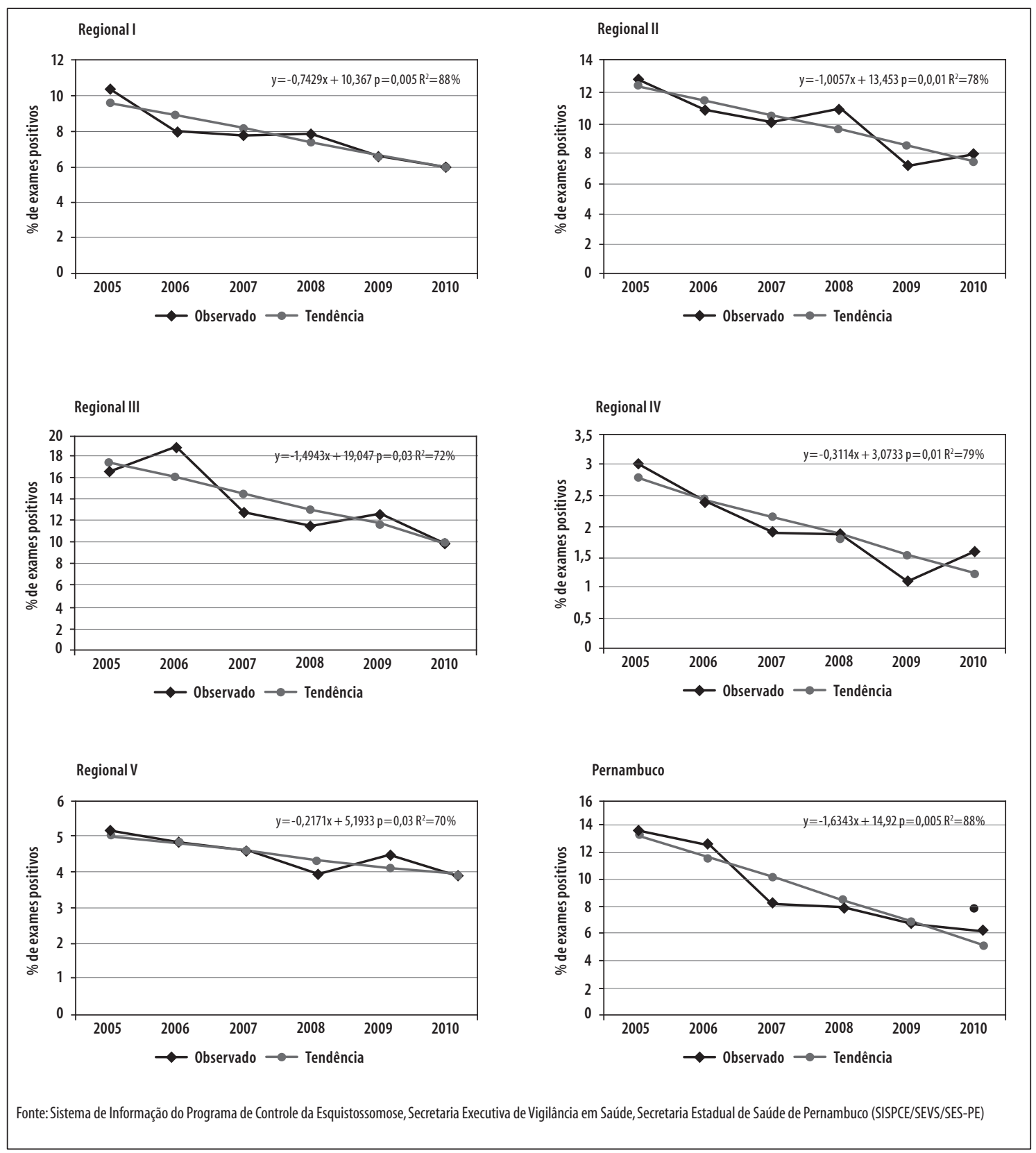

\section{Figura 1 - Tendência de declínio do percentual de exames laboratoriais positivos para a esquistossomose por Regionais de Saúde e pelo conjunto do estado de Pernambuco, 2005 a 2010}

Dados do Ministério da Saúde apontam a região Nordeste do Brasil como responsável pelo maior registro de casos de esquistossomose e situam Pernambuco entre os estados com prevalência média mais elevada de pessoas infectadas pelo $S$. mansoni. Considera-se a endemia uma das principais causas de mortalidade por doença transmissível. ${ }^{3}$
Condições ambientais, como a presença de rios e clima favorável para reprodução de caramujos do gênero Biomphalaria, ${ }^{10}$ e condições socioeconômicas, como o saneamento básico precário, difícil acesso a atendimento médico e acentuada pobreza ${ }^{11}$ contribuem para a manutenção do ciclo de transmissão da esquistossomose no estado. 
Em Pernambuco, a esquistossomose é historicamente endêmica na zona rural, apresentando áreas com até $80 \%$ de indivíduos parasitados. ${ }^{12,13}$ Inquéritos realizados em escolares na faixa etária de 9 a 12 anos, residentes em municípios situados na Zona da Mata do estado, região de localização dos municípios das RS II e III, demonstraram a ocorrência de transmissão recente da parasitose nessa área. ${ }^{14,15}$

No inquérito realizado por Barbosa ${ }^{14,15}$ em 2005, quase metade (45,5\%) dos municípios localizados na área que compõe a RS II apresentou positividade considerada moderada (10 a 50\%) para esquistossomose e a grande maioria (73\%) dos municípios localizados na área correspondente à RS III apresentou a mesma positividade. Os resultados apresentados neste trabalho mostram concordância com os resultados dos estudos supracitados e reforçam a permanência da Zona da Mata de Pernambuco como a área de maior transmissão da doença, apresentando a RS III como a de maior positividade nos anos analisados, seguida pela RS II. A região da Zona da Mata apresenta condições ambientais favoráveis, extensa coleção hídrica e grande presença de criadouros de hospedeiros intermediários, viabilizando a sobrevivência do parasito fora do hospedeiro humano. Ademais, com a manutenção da pobreza, os baixos níveis de higiene e de saneamento básico favorecem a permanência do ciclo de transmissão da doença nessa área.

0 presente trabalho observou que a RS I (Recife) foi a terceira de maior positividade para esquistossomose no estado. A RS I é composta, em grande parte, por municípios da Região Metropolitana do Recife, incluindo áreas litorâneas. 0 rápido e desordenado processo de migração e urbanização verificado em Pernambuco contribuiu para a expansão da endemia. Diferentes estudos, realizados em áreas litorâneas do estado, têm revelado mudança no perfil epidemiológico da esquistossomose, com a detecção de casos de infecção aguda e crônica nessas áreas. ${ }^{16-20} 0$ deslocamento de indivíduos infectados de áreas endêmicas para áreas consideradas sem transmissão, como as periferias das grandes cidades, e a ocupação humana dessas áreas de forma desorganizada, sob precárias condições - básicas - de higiene, saneamento e infraestrutura, contribuíram para a contaminação de ambientes aquáticos, criadouros de caramujos, pela presença de fezes contendo ovos de $S$. mansoni e, por conseguinte, o surgimento de novos focos da doença. ${ }^{18}$
A redução na presença da doença no Estado é evidente ao se comparar os resultados deste estudo com inquéritos já realizados, referentes a estudos anteriores. ${ }^{13,21,22}$ A implementação do PCE em nível local, com a busca de portadores e tratamento dos casos positivos, tem contribuído para a redução da endemia no estado, refletida na diminuição dos casos de hospitalização por esquistossomose em Pernambuco. ${ }^{23}$

Outros estudos, também baseados em dados secundários obtidos do SISPCE, mostraram resultados similares aos encontrados neste trabalho, com redução da positividade em outros estados do Nordeste considerados endêmicos para a esquistossomose. Em Sergipe, foi verificada uma redução na ocorrência da doença: de 13,6\%, em 2005, para 10,6\%, em 2008. ${ }^{24}$ No estado do Maranhão, uma análise dos casos de esquistossomose entre 1997 e 2003 apontou tendência decrescente em duas das quatro RS analisadas. ${ }^{25}$ Análise do percentual de positividade da doença no Brasil, realizada pelo Ministério da Saúde sobre a série histórica de 1990 a 2010, mostrou o mesmo panorama de redução com positividade média de $8 \%$ e uma tendência de decréscimo de $0,25 \%$ ao ano. ${ }^{3}$

Embora o estado de Pernambuco, de modo geral, apresente tendência de redução da positividade nos exames coproscópicos realizados, vale ressaltar a dinâmica de transmissão focal da esquistossomose em áreas restritas, internas aos municípios pernambucanos, com elevado número de indivíduos positivos e prevalência da doença de até $70 \% .{ }^{11}$ Dessa forma, é necessária, também, a análise da positividade por municípios e localidades para verificar se a esquistossomose nessas áreas também apresenta tendência de redução na positividade de exames coproscópicos. Outro ponto importante a se considerar é o fato de todavia, não se haver reduzido a prevalência até valores inferiores a 5\%, conforme recomenda a Organização Mundial da Saúde (OMS) ${ }^{26}$

Importante salientar que, no período analisado, observou-se uma redução bastante expressiva no quantitativo de exames realizados em populações dos municípios endêmicos da RS III (Palmares), com possível impacto na redução do total do número de exames realizados em Pernambuco: a diminuição na busca por novos casos da doença pode ter influenciado na diminuição da positividade para esquistossomose no estado. Não obstante essa redução no número de 
exames, a RS III continuou a apresentar positividade para a doença acima das demais Regionais, em todos os anos analisados. Entretanto, a redução do número de exames da RS III não invalida a tendência de redução da positividade da esquistossomose nas outras RS.

Quinino e colaborados analisaram a cobertura de tratamento para esquistossomose em dois estudos distintos. 0 primeiro estudo, ${ }^{27}$ realizado nos municípios da Região Metropolitana do Recife (RS I), em sete dos 11 municípios analisados, apresentou baixas coberturas entre os anos de 2003 e 2005.0 segundo estudo, ${ }^{28}$ conduzido em municípios de abrangência das RS II e III, verificou que metade deles apresentava coberturas abaixo de $80 \%$, de acordo com o preconizado pelo MS. Os resultados do presente estudo mostraram a permanência de coberturas de tratamento abaixo do preconizado nas RS I (Recife) e II (Limoeiro).

A ausência de tratamento oportuno dos casos positivos de esquistossomose na maioria dos municípios pode ser resultante da escassez de agentes nas atividades de rotina do PCE, que não conseguem corresponder à demanda de diagnóstico e tratamento necessários, seja pela falta de encaminhamento dos casos positivos às unidades de saúde, seja pela dificuldade de acesso da população ao tratamento com praziquantel nas unidades de saúde, e até pela resistência em realizar o tratamento devido aos possíveis efeitos adversos provocados pelo medicamento. A baixa cobertura de tratamento contribui para a manutenção de focos de transmissão e indivíduos sem tratamento adequado são potenciais portadores das formas crônicas e graves da doença.

Mesmo que o uso de dados secundários seja apontado como uma limitação deste estudo, uma vez sujeitos a vieses relacionados à digitação incorreta dos dados nos municípios, a existência de um sistema de informações (SISPCE) permite a construção dos indicadores de positividade e cobertura de tratamento, e sendo assim, a possibilidade de seu uso adequado e regular como se pretendeu nesta avaliação. Apesar das dificuldades operacionais, o SISPCE representa um avanço no monitoramento e vigilância da esquistossomose. Ademais, o fato de o PCE realizar o diagnóstico parasitológico nos municípios pelo método Kato-Katz, com apenas uma lâmina para cada indivíduo, pode resultar em casos falso-negativos devido à ausência de ovos do $S$. mansoni no início da infecção ou logo após o tratamento medicamentoso, à baixa carga parasitária e à inconsistência de eliminação dos ovos pelo parasito.

Segundo a OMS, a eliminação da esquistossomose é possível. Vários países endêmicos, a exemplo da Tunísia, não têm relatado um único caso novo de esquistossomose em anos e caminham no sentido da validação da interrupção da transmissão do parasito. Outros países, como o Marrocos, têm conseguido baixar a transmissão da doença e procura definir diretrizes, estratégias e instrumentos para avaliar a interrupção da transmissão. ${ }^{29}$ Ainda de acordo com a OMS, os países das Américas têm progredido na redução da transmissão da doença, ${ }^{29}$ situação fortalecida com os resultados observados ao longo dos anos e analisados no presente estudo. Entretanto, resta um longo caminho a ser percorrido para 0 alcance da eliminação da esquistossomose mansoni como problema de Saúde Pública no Brasil. Faz-se necessária uma intensificação das ações de controle, aliada às melhorias sanitárias, para oferecer diagnóstico e tratamento oportunos à população das áreas endêmicas.

Em 2011, a Secretaria Estadual de Saúde de Pernambuco, por meio do Programa Sanar, priorizou 0 enfrentamento às doenças negligenciadas com 0 propósito de realizar ações direcionadas à redução da carga e/ou eliminação de sete doenças negligenciadas. Para a esquistossomose, o Sanar elegeu 40 municípios prioritários pelo critério de uma média anual de prevalência maior ou igual a $10 \%$ entre os anos de 2005 e 2010, e estabeleceu como meta a redução da prevalência da doença para menos de 10\% nesses municípios, até 2014. As ações do Programa Sanar dirigidas ao enfrentamento da esquistossomose têm por princípio fortalecer o envolvimento das equipes de saúde da Atenção Básica com a Vigilância, visando aumentar a captação de novos casos e a cobertura de tratamento dos casos positivos, além do tratamento coletivo da população de localidades hiperendêmicas em Pernambuco. Ao fim das ações desenvolvidas pelo Sanar, os resultados alcançados fornecerão um panorama que permita comparar o perfil epidemiológico da esquistossomose antes e depois das medidas de intervenção em Pernambuco, e verificar o impacto ocasionado pela intensificação das ações de controle nos municípios que apresentavam a maior carga da doença no estado. 
O Brasil tem como objetivo eliminar a esquistossomose como problema de Saúde Pública nos municípios endêmicos. ${ }^{3}$ Nesse caminho, para que as metas estabelecidas pela OMS e pelo governo brasileiro sejam alcançadas, é necessária a intensificação das ações de vigilância e controle do PCE nas áreas endêmicas do estado pernambucano, refletida no diagnóstico e tratamento oportunos dos casos de esquistossomose. A priorização no enfretamento dessa doença contribuirá para a mudança do perfil epidemiológico, reduzindo, cada vez mais, o número de casos de esquistossomose e, consequentemente, a ocorrência das formas graves e a mortalidade a ela associada, e possibilitando alcançar os níveis de controle e eliminação da esquistossomose no estado.

Pernambuco apresentou tendência decrescente e constante na ocorrência de casos de esquistossomose em suas Regionais de Saúde situadas na área endêmica, ao longo do período analisado. É importante o contínuo monitoramento da positividade e da distribuição dessa endemia, assim como o desenvolvimento de análises que considerem os municípios e localidades com a maior carga da doença, para verificação de sua tendência nos anos seguintes e mensuração do impacto das intervenções e políticas públicas voltadas ao controle da esquistossomose.

\section{Referências}

1. Ministério da Saúde (BR). Secretaria de Vigilância em Saúde. Departamento de Vigilância Epidemiológica. Guia de vigilância epidemiológica. 7. ed. Brasília: Ministério da Saúde; 2009.

2. Pan-American Health Organization. Epidemiological profiles of neglected diseases and other infections related to poverty in Latin America and the Caribbean [Internet]. Washington: Pan-American Health Organization; 2009 [cited 2014 Mar 31]. Available from: http://www2.paho.org/hq/dmdocuments/2009/ nds-epi-profiles.pdf

3. Ministério da Saúde (BR). Secretaria de Vigilância em Saúde. Departamento de Vigilância em Doenças Transmissíveis. Plano integrado de ações estratégicas de eliminação da hanseníase, filariose, esquistossomose e oncocercose como problema de saúde pública, tracoma como causa de cegueira e controle das geo-helmintíases: plano de ação 20112015. Brasília: Ministério da Saúde; 2012.

4. Pordeus LC, Aguiar LR, Quinino LRM, Barbosa CS. A ocorrência das formas aguda e crônica da esquistossomose mansônica no Brasil no período de

\section{Agradecimentos}

À Secretaria de Executiva de Vigilância em Saúde da Secretaria Estadual de Saúde de Pernambuco, pela cessão de informações referentes aos dados contidos no Sistema de Informação do Programa de Controle da Esquistossomose - SISPCE.

À Roselene Hans, diretora geral de Controle de Doenças e Agravos, e à Bárbara Morgana da Silva, gerente de Vigilância e Controle de Doenças Negligenciadas Transmitidas por Vetores, da Secretaria Estadual de Saúde de Pernambuco, pelo apoio ao desenvolvimento desta pesquisa.

\section{Contribuição das autoras}

Barreto AVMSB participou da análise e intepretação dos dados, redação e revisão do conteúdo do manuscrito.

Melo ND, Torres JV e Santiago RT participaram da concepção e delineamento do estudo, coleta e análise dos dados e redação do conteúdo do manuscrito.

Silva MBA participou da concepção e delineamento do estudo e da revisão crítica do conteúdo intelectual do manuscrito.

Todos os autores aprovaram a versão final do manuscrito e declaram serem responsáveis por todos os aspectos do trabalho, garantindo sua precisão e integridade.

1997 a 2006: uma revisão de literatura. Epidemiol Serv Saude. 2008 jul-set;17(3):163-75.

5. Ministério da Saúde (BR). Secretária de Vigilância em Saúde. Sistema nacional de vigilância em saúde: relatório de situação: Pernambuco. 5. ed. Brasília: Ministério da Saúde; 2011.

6. Ministério da Saúde (BR). Secretaria de Atenção à Saúde. Departamento de Atenção à Saúde. Vigilância em saúde: dengue, esquistossomose, hanseníase, malária, tracoma e tuberculose. 2. ed. Brasília: Ministério da Saúde; 2008. (Cadernos de atenção básica; $n^{0} 21$ ).

7. Secretaria Estadual de Saúde (Pernambuco). Secretaria Executiva de Vigilância em Saúde. Programa de Enfretamento das Doenças Negligenciadas no Estado de Pernambuco/SANAR - 2011-2014 [Internet]. 2. ed. Recife: Secretaria Estadual de Saúde; 2013 [citado 2013 dez 5]. Disponível em: http://portal.saude. pe.gov.br/sites/portal.saude.pe.gov.br/files/plano_ sanar_2011-2014.pdf

8. Instituto Brasileiro de Geografia e Estatística. Sinopse do censo demográfico 2010: Pernambuco 
[Internet]. 2010 [citado 2013 dez 5]. Disponível em: http://www.censo2010.ibge.gov.br/sinopse/index. php?uf $=26 \&$ dados $=0$

9. Ministério da Saúde (BR). Secretaria de Vigilância em Saúde. Situação epidemiológica da esquistossomose no Brasil: grupo técnico das parasitárias, sub HA/ CGDT/DEVEP/SVS/MS. Brasília: Ministério da Saúde; 2010.

10. Ministério da Saúde (BR). Secretaria de Vigilância em Saúde. Departamento de Vigilância Epidemiológica. Vigilância e controle de moluscos de importância epidemiológica. Diretrizes técnicas: Programa de Vigilância e Controle da Esquistossomose (PCE). 2. ed. Brasília: Ministério da Saúde; 2007.

11. Secretaria Estadual de Saúde (Pernambuco). Secretaria Executiva de Vigilância em Saúde. Esquistossomose e geo-helmintíases: relatório das condições de saneamento das áreas/localidades hiperendêmicas em Pernambuco [Internet]. Recife: Secretaria Estadual de Saúde; 2013 [citado 2013 nov 10]. Disponível em: http://portal.saude.pe.gov. br/sites/portal.saude.pe.gov.br/files/relatorio_das_ condicoes_de_saneamento_das_localidades_ hiperendemicas_em_pe.pdf

12. Coutinho EM, Abath FG, Barbosa CS, Domingues AL, Melo MC, Montenegro SM, et al. Factors involved in Schistosoma mansoni infection in rural areas of Northeast Brazil. Mem Inst Oswaldo Cruz. 1997 SepOct;92(5):707-15.

13. Favre TC, Ximenes RA, Galvão AF, Pereira AP, Wanderlei TN, Barbosa CS, et al. Reliability of current estimates of schistosomiasis prevalence in the Rainforest Zone of the state of Pernambuco, Northeastern Brazil. Mem Inst Oswaldo Cruz. 2006 Sep;101 Suppl 1:73-8.

14. Barbosa CS, Favre TC, Wanderley TN, Callou AC, Pieri OS. Assessment of schistosomiasis, through school surveys, in the Forest Zone of Pernambuco, Brazil. Mem Inst Oswaldo Cruz. 2006 Oct;101 Suppl 1:5-62.

15. Barbosa VS, Araújo KC, Leal Neto OB, Barbosa CS. Spatial distribution of schistosomiasis and geohelminthiasis cases in the rural areas of Pernambuco, Brazil. Rev Soc Bras Med Trop. 2012 Oct;45(5):633-8.

16. Barbosa CS, Pieri OS, Silva CB, Barbosa FS Ecoepidemiologia da esquistossomose urbana na ilha de Itamaracá, Estado de Pernambuco. Rev Saude Publica. 2000 ago;34(4):337-41.

17. Barbosa CS, Domingues ALC, Abath F, Montenegro SML, Guida U, Carneiro J, et al. Epidemia de esquistossomose aguda na praia de Porto de Galinhas, Pernambuco, Brasil. Cad Saude Publica. 2001 maijun;17(3):725-8.

18. Barbosa CS, Araújo KC, Sevilla MA, Melo F, Gomes EC, Souza-Santos R. Current epidemiological status of schistosomiasis in the state of Pernambuco, Brazil. Mem Inst Oswaldo Cruz. 2010 Jul;105(4):549-54.

19. Barbosa CS, Leal-Neto OB, Gomes ECS, Araújo KCGM, Domingues ALC. The endemisation of schistosomiasis in Porto de Galinhas, Pernambuco, Brazil, 10 years after the first epidemic outbreak. Mem Inst Oswaldo Cruz. 2011 Nov;16(7):878-83.

20. Barbosa CS, Barbosa VS, Melo FL, Melo MSB, Bezerra L, Campos JV, et al. Casos autóctones de esquistossomose mansônica em crianças de Recife, PE. Rev Saude Publica. 2013 ago;47 (4):684-90.

21. Barbosa CS, Silva CB, Barbosa FS. Esquistossomose: reprodução e expansão da endemia no Estado de Pernambuco no Brasil. Rev Saude Publica. 1996 dez;30(6):609-16.

22. Coura JR, Amaral RS. Epidemiological and control aspects of schistosomiasis in Brazilian endemic areas. Mem Inst Oswaldo Cruz. 2004 ago;99 Suppl $1: 13-9$.

23. Resendes APC, Souza-Santos R, Barbosa CS. Hospitalization and mortality from mansoni schistosomiasis in the State of Pernambuco, Brazil, 1992/2000. Cad Saude Publica. 2005 SepOct;21(5):1392-401.

24. Rollemberg CV, Santos CMB, Silva MMBL, Souza AMB, Silva AM, Almeida JA, et al. Aspectos epidemiológicos e distribuição geográfica da esquistossomose e geo-helmintos, no Estado de Sergipe, de acordo com os dados do Programa de Controle da Esquistossomose. Rev Soc Bra Med Trop. 2011 jan-fev;44(1):91-6.

25. Cantanhede SPD, Ferreira AP, Mattos IE. Esquistossomose mansônica no Estado do Maranhão, Brasil, 1997-2003. Cad Saude Publica. 2011 abr;27(4):811-6.

26. World Health Organization. Schistosomiasis: progress report 2001-2011 and strategic plan 2012-2020. Geneva: WHO Library; 2013.

27. Quinino LRM, Costa JMBS, Aguiar LR, Wanderley TNG, Barbosa CS. Avaliação das atividades de rotina do Programa de Controle da Esquistossomose em municípios da Região Metropolitana do Recife, Pernambuco, entre 2003 e 2005. Epidemiol Serv Saude. 2009 dez;18(4):335-43. 
28. Quinino LRM, Barbosa CS, Samico L. O programa de controle da esquistossomose em dois municípios da zona da mata de Pernambuco: uma análise de implantação. Rev Bra Saude Mater Infant. 2010 nov;10(1):119-29.

29. World Health Organization. Elimination of schistosomiasis in low transmission areas. Report of the WHO informal consultation [Internet]. 2008 Aug 18-19; Salvador, BA. Geneva: World Health Organization; 2009 [cited 2014 Mar 31]. Available from: whqlibdoc.who.int/hq/2009/WHO_HTM_NTD_ PCT.2009.2_eng.pdf

Recebido em 11/06/2014

Aprovado em 07/12/2014 